\title{
Updates in neurosonography
}

\section{Corinne Veyrac}

(C) Springer-Verlag 2011

While MRI is the gold standard for imaging the brain, US remains a sensitive first-line test for detecting brain damage in the neonate. It is widely used for repeat bedside imaging in the intensive care unit, because a great number of infants, e.g., neonates with extremely low birth weights, cannot be moved for an MRI scan.

The sensitivity of US depends on the scan technique used. Besides using a convex probe for imaging the entire brain through the traditional anterior fontanel approach, several supplementary techniques should routinely be added to any brain US examination.

1. Linear high-frequency probes permit an accurate analysis of the parenchyma and extra-axial spaces for enhanced:

- detection of superficial cortical echodense ischaemic damage

- demonstration of a cortical and/or subcortical lesion and its perilesional oedema

- recognition of the changing echostructure of a peripheral lesion, such as the development of tiny cysts within an ischaemic grey and/or white matter damage, or the liquefaction of the superficial cortical layer deep to a subpial haemorrhage

- detection of abnormal gyration or grey/white matter organisation

- location of an extra-axial collection, determining its echodensity and mass effect.

Disclaimer DR. Veyrac has no financial interest, investigational or off-label uses to disclose.

C. Veyrac $(\square)$

Service de Radiologie Pédiatrique, Hôpital Arnaud de Villeneuve, Montpellier, France

e-mail: c-veyrac@chu-montpellier.fr
2. The use of accessory acoustic windows permits displaying regions that are beyond the reach via the anterior fontanel:

- the mastoid fontanel for imaging the posterior fossa and cerebral peduncles, allowing detection and evaluation of cerebellar haematoma, and appreciation of the fourth ventricle volume and content in case of intraventricular haemorrhage

- the posterior fontanel for demonstrating the echodensity of the parenchyma posterior to the lateral ventricles, especially in case of severe ventricular dilatation; detecting hyperechogenic haemorrhagic spots, small cysts of periventricular leucomalacia, etc.

- any cranial suture near peripheral lesions, allowing an accurate analysis with high-frequency probes, and distinguishing a parenchymal from an extraaxial location.

As for the conventional acoustic window, highfrequency transducers may add valuable information to these alternative views.

3. The routine use of colour Doppler permits not only guidance for pulsed Doppler measurements, but also:

- detection of flow of cerebrospinal fluid within the aqueduct of Sylvius, confirming intraventricular haemorrhage in equivocal cases; or demonstrating ventriculitis before a microbiological diagnosis is available, or during the follow-up in septic meningitis

- demonstration of the patency of the internal cerebral vein and some venous sinuses, and of the terminal veins in patients with severe intraventricular haemorrhage. Terminal vein patency 
reduces the probability of subsequently developing ischaemic-haemorrhagic infarction.

4. The use of pulsed Doppler may be helpful for:

- predicting increased risk of intraventricular haemorrhage or ischaemic brain damage during examinations performed early after birth by showing extremely low or extremely high cerebral blood flow velocities

- following posthaemorrhagic or postmeningitis progressive ventricular dilatation by recognising a significant increase in resistive indices, which indicate raised intracranial pressure
- evaluating the haemodynamic status of a vein of Galen aneurysm.

In brain US, pulsed Doppler only provides information supplementary to the morphological investigation. Therapeutic decisions are based on a combination of clinical, electroencephalography (EEG), and US greyscale and Doppler findings. US is not in competition with MRI. These are complementary imaging tools that should be integrated in the management of a neonate, and should follow clear protocols: (1) US for screening, (2) MRI for complete evaluation of the brain, (3) US for the early follow-up and (4) MRI for long-term follow-up. 